\title{
Rheumatology
}

\section{Percutaneous Coronary Intervention Outcomes in Patients with Rheumatoid Arthritis, Systemic Lupus Erythematosus and Systemic sclerosis}

\begin{tabular}{|c|c|}
\hline Journal: & Rheumatology \\
\hline Manuscript ID & RHE-19-1694.R1 \\
\hline Manuscript Type: & Original Article \\
\hline $\begin{array}{r}\text { Date Submitted by the } \\
\text { Author: }\end{array}$ & 20-Nov-2019 \\
\hline Complete List of Authors: & $\begin{array}{l}\text { Martinez, Sara; Providence St. Peter Hospital, Cardiology } \\
\text { Mohamed, Mohamed; Keele University, Cardiology } \\
\text { Potts, Jessica; Keele University, Cardiology } \\
\text { Abhishek, Abhishek; City Hospital, Academic Rheumatology } \\
\text { Roddy, Edward; Keele University, School of Primary, Community and } \\
\text { Social Care } \\
\text { Savage, Michael; Thomas Jefferson University, Cardiology } \\
\text { Bharadwaj, Aditya; Loma Linda University Medical Center, Cardiology } \\
\text { Kwok, Chun Shing; Keele University, Cardiology } \\
\text { Bagur, Rodrigo; Keele University, Cardiology } \\
\text { Mamas, Mamas; Keele University, Cardiology }\end{array}$ \\
\hline $\begin{array}{r}\text { Keywords }<\text { br }>\text { Please select a } \\
\text { minimum FIVE keywords from } \\
\text { the list provided. These } \\
\text { keywords will be used to } \\
\text { select reviewers for this } \\
\text { manuscript. The keywords in } \\
\text { the main text of your paper } \\
\text { do not need to match these } \\
\text { words.: }\end{array}$ & $\begin{array}{l}\text { Rheumatoid arthritis < RHEUMATIC DISEASES, Scleroderma and related } \\
\text { disorders < RHEUMATIC DISEASES, Cardiovascular < TISSUES, } \\
\text { Epidemiology < BASIC \& CLINICAL SCIENCES, Statistics < } \\
\text { MISCELLANEOUS }\end{array}$ \\
\hline
\end{tabular}




\section{Percutaneous Coronary Intervention Outcomes in Patients with Rheumatoid Arthritis, Systemic Lupus Erythematosus and Systemic sclerosis}

Short Title: PCI outcomes in rheumatic disease.

Sara C. Martinez*1, Mohamed Mohamed ${ }^{2,3}$, Jessica Potts ${ }^{2}$, Abhishek Abhishek ${ }^{4}$, Edward Roddy $^{5}$, Michael Savage ${ }^{6}$, Aditya Bharadwaj ${ }^{7,8}$, Chun Shing Kwok ${ }^{2,3}$, Rodrigo Bagur ${ }^{1}$, Mamas A. Mamas 2,3

(1) Division of Cardiology, Providence St. Peter Hospital, Olympia, Washington, USA

(2) Keele Cardiovascular Research Group, Centre for Prognosis Research, Keele University, UK

(3) Royal Stoke University Hospital, Stoke-on-Trent, UK

(4) Academic Rheumatology, University of Nottingham, Nottingham, UK

(5) School of Primary, Community and Social Care, Keele University, UK

(6) Haywood Academic Rheumatology Centre, Midland Partnership NHS Foundation Trust, Haywood Hospital, Burslem, UK.

(7) Department of Medicine (Cardiology), Thomas Jefferson University Hospital, Philadelphia, Pennsylvania, United States

(8) Cardiology, Loma Linda University, California, United States

*These authors contributed equally - joint first authors

Correspondence to:

Prof. Mamas A. Mamas

Professor of Cardiology

Keele Cardiovascular Research Group,

Centre for Prognosis Research,

Institute for Primary Care and Health Sciences,

Keele University, UK

mamasmamas1@yahoo.co.uk

Word count (inc. title page, abstract and references): 5200 


\begin{abstract}
Objective: Patients with autoimmune rheumatic disease (AIRD) are at an increased risk of coronary artery disease. The present study sought to examine the prevalence and outcomes of AIRD patients undergoing percutaneous coronary intervention (PCI) from a national perspective.

Methods: All PCI-related hospitalizations recorded in the United States (US) National Inpatient Sample (2004 to 2014) were included, stratified in to 4 groups: No AIRD, rheumatoid arthritis (RA), systemic lupus erythematosus (SLE) and systemic sclerosis (SSC). We examined the prevalence of AIRD subtypes and assessed their association with in-hospital adverse events using multivariable logistic regression (odds ratios (OR) [95\% confidence interval]).
\end{abstract}

Results: Patients with AIRD represented 1.4\% $(n=90,469)$ of PCI hospitalizations. The prevalence of RA increased from $0.8 \%$ (2004) to $1.4 \%$ (2014), but other AIRD subtypes remained stable. In multivariable analysis, the adjusted odds of in-hospital complications (aOR any complication: 1.13 [1.01-1.26]; all-cause mortality:1.32 [1.03-1.71]; bleeding: 1.50 [1.301.74]; stroke: 1.36 [1.14-1.62]) were significantly higher in patients with SSC compared to those without AIRD. There was no difference in complications between SLE and RA groups and those without AIRD, except higher odds of bleeding in SLE patients (aOR 1.19 [1.091.29]) and reduced odds of all-cause mortality in RA patients (aOR 0.79 [0.70-0.88]).

Conclusion: In a nationwide cohort of US hospitalizations, we demonstrate increased rates of all adverse clinical outcomes following PCI in people with SSC, and increased bleeding in SLE. Management of such patients should involve a multi-team approach with rheumatologists.

Key Words: percutaneous coronary intervention, rheumatoid arthritis, systemic sclerosis, systemic lupus erythematosus, outcomes. 


\section{Key messages:}

- This is the first study to report prevalence, characteristics and outcomes of AIRD patients undergoing PCI.

- The prevalence of RA increased over 11 years, with no change in SLE and SSC undergoing PCI

- PCI outcomes differ according to AIRD subtype, with worse clinical outcomes in patients with SSC.

\section{Introduction}

Cardiovascular disease (CVD) remains the leading cause of death for patients in the United States, with patients with chronic inflammatory conditions at particular risk of CVD.[14] The most common form of CVD is coronary artery disease (CAD) which is more prevalent in patients with autoimmune rheumatic diseases (AIRD) such as rheumatoid arthritis (RA), systemic lupus erythematosus (SLE) and systemic sclerosis (SSC). This population has a $>1.5$ fold increased risk of short-term cardiovascular mortality. $[\underline{1}, \underline{2}, \underline{5-7}]$ Several factors place patients with AIRD at a heightened risk of coronary artery disease such as accelerated atherosclerosis and inflammation, making coronary plaques more vulnerable to rupture and subsequent thrombosis.[] $]$

Percutaneous coronary intervention (PCI) is the most common modality of revascularization in patients with significant $\mathrm{CAD}$. The majority of outcomes data on patients with AIRD undergoing PCI are from small studies and case reports.[9-12] Despite revascularization in these patients, they will remain at a further risk of future ischemic events and disease progression, [13] which is often more aggressive than in patients without AIRD.[7] Few studies have examined the outcomes of PCI in patients with common AIRDs such as RA and SLE, but they were limited by their small sample sizes or highly selected cohorts from 
individual centers and, therefore, insufficiently powered to detect any clinically significant differences.

The present study sought to examine the prevalence and in-hospital clinical outcomes of patients with AIRD undergoing PCI in a national cohort of hospitalizations in the United States (US) over an eleven-year period.

\section{Methods}

\section{Data Source}

The study was undertaken in the National Inpatient Sample (NIS) for hospital discharges in the United States between 2004 and 2014. The NIS is the largest all-payer inpatient health care database in the United States and was developed by the Healthcare Cost and Utilization Project (HCUP), which is sponsored by the Agency for Healthcare Research and Quality (AHRQ). [14] The NIS dataset contains hospital information on 7 to 8 million hospital discharges per year from 2004 onwards. The sampling strategy has changed over time in order to produce more generalizable estimates by reducing sampling bias. Before 2012 the NIS retained all discharges, but only from a sample of hospitals. Since the redesign, the NIS now samples discharges from all hospitals participating in HUCP, approximating a 20\% stratified sample of all discharges from US community hospitals.

\section{Study Design and Population}

We identified all individuals who had undergone a PCI between January 2004 and December 2014 by identifying all eligible discharges with an International Classification of Diseases, Ninth Revision, Clinical Modification (ICD-9-CM) procedure code of 00.66 (Percutaneous Transluminal Coronary Angioplasty), 36.06 (Insertion of non-drug-eluting coronary artery stent(s)) or 36.07 (Insertion of a drug-eluting coronary artery stent(s)). Before a revision of the codes in 2005 the codes 36.01 (Single vessel percutaneous transluminal 
coronary angioplasty or coronary atherectomy without mention of thrombolytic agent), 36.02 (Single vessel percutaneous transluminal coronary angioplasty or coronary atherectomy with mention of thrombolytic agent) and 36.05 (Multiple vessel percutaneous transluminal coronary angioplasty [PTCA] or coronary atherectomy performed during the same operation, with or without mention of thrombolytic agent) were also used and so these codes were also included when identifying procedures in discharges from 2004 and 2005.

All records were eligible for inclusion as long as the discharge record showed that the patient had undergone a PCI procedure during their hospital stay and was over the age of 18 years. Information of patient demographics is recorded for each hospital discharge including data regarding age, gender, race, admission type (elective or emergent), admission day (weekday or weekend), median household income according to ZIP code and expected primary payer. Each discharge record had information on up to 30 diagnoses that the patient had been given (15 between 2004 and 2008, 25 between 2009 and 2013 and 30 in 2014), and it was these diagnosis codes that were used to identify records with a diagnosis of RA (714.0), SLE (710.0) or SSC (710.1). Information was also collected on the long-term use of steroids, V58.65. All records with more than one AIRD were excluded ( $n=2499,0.04 \%$ of entire cohort), as were any records with less than $10 \%$ missing data (missing age and primary expected payer, $\mathrm{n}=9589$; $0.15 \%$ of the entire cohort).

Finally, information about the PCI procedure was determined from the procedure codes, including whether the PCI was a multi vessel, single vessel and whether it involved bifurcation stenting. The use of adjunctive devices including intracoronary pressure wire, intravascular ultrasound and an assist device (such as an intra-aortic balloon pump) were also recorded. Where available from the procedure codes we also included the type of stent deployed (bare metal, drug-eluting). 


\section{Outcomes}

In-hospital clinical outcomes and complications were identified. The main outcomes chosen included: (a) in-hospital all-cause mortality, (b) and in-hospital complication or (c) a composite of any of the in-hospital complications considered. The length of stay and the total charge of hospitalization were also considered. The total charge given in the dataset represents the amount that hospital billed for the services, but is not representative of the hospital services actually cost. Therefore, a charge to cost conversion ratio was used in order to covert the reported charges into the actual cost for the payer.

Procedural complications were also identified using ICD-9-CM codes and patient safety indicators including post-operative hemorrhage requiring transfusion, vascular complications, cardiac complications including iatrogenic and pericardial complications, whether an individual required bail out or emergency coronary artery bypass grafting and postoperative stroke or transient ischemic attack. Finally, bleeding complications were identified, including gastrointestinal, retroperitoneal, intracranial, intracerebral hemorrhage, unspecified hemorrhage, and whether a blood transfusion was required. Complications were identified by ICD-9-CM codes in any secondary diagnosis field (Supplementary Table S1, available at Rheumatology online).

\section{Statistical Analysis}

Statistical analysis was performed on Stata/MP version 14.0. Continuous variables are presented as median and interquartile range, and categorical data are presented as number and percentage. For all analyses, the survey estimation commands were used (by using the svy prefix in analyses conducted in Stata), this followed the recommendations from AHRQ for analysis of survey data to account for the complex survey design of the NIS database. As records were not sampled individually but by hospital number, clustering of records within 
hospitals was taken into account in the survey estimation. This was done by defining each hospital to be the primary sampling unit. For calculation of national estimates and correct variances, sampling weights for each individual discharge that were provided by the AHRQ were used. The use of sampling weights is required because the design of the study means that different observations may have different probabilities of selection. Due to the redesign of the NIS data and the alternative sampling strategy used before 2012, these weights needed to be updated from the original sampling weights for 2004-2011 in order for the analysis to be conducted across all included years.

Multivariable logistic regression models were fitted using maximum likelihood estimation in order to investigate the association of rheumatological diagnoses (RA, SLE, SSC) with odds of a) in-hospital death, b) in-hospital PCI complications, including procedure-related bleeding, vascular complication, cardiac complication or stroke/TIA, and c) the composite of any complication, compared to patients without AIRD, adjusting for potential confounders in all models. Confounders included age, gender, ethnicity, median income, primary payer, elective admission, day of admission (weekend/weekday) primary diagnosis of MI, any diagnosis of STEMI/NSTEMI during admission, diagnosis of shock, hypercholesterolemia, patient smoking status, Elixhauser comorbidities, use of an assist device or IABP, use of a bare metal or drug eluting stent, bifurcation stenting, fractional flow reserve, single or multi-vessel PCI and year of hospitalization, as well as accounting for the clustering of individuals by hospital. Confounders measured are presented in Table 1.

A subgroup analysis was conducted to compare the outcomes of patients with RA with and without long-term steroid use.

\section{Results}


A total of 6,558,947 hospitalizations for PCI procedures were recorded in U.S. hospitals between 2004 and 2014, of which 90,469 patients (1.4\%) had a rheumatological diagnosis. The percentages of patients with RA, SLE and SSC were: $1.06 \%(n=69,354), 0.27 \%(n=17,661)$ and $0.05 \%(n=3,454)$, respectively (Table 1$)$. There was an increase in the number of patients with RA receiving PCI over the study period $(0.8 \%$ in 2004 to $1.4 \%$ in $2014, \mathrm{p}<0.001$ for trend) (Figure 1), while SLE and SSC remained stable (2004 to $2014: 0.2 \%$ to $0.4 \%, p=0.09$, and $0.1 \%$ to $0.1 \%, p=0.34$, respectively).

Several differences were observed between the study groups. Women comprised the majority of PCI cases for RA, SLE, and SSC at 56\%, 78\%, and 74\%, respectively, compared to only $33.3 \%$ in cases without these conditions. (Table 1) Patients with RA/SLE/SSC were more likely to have a primary diagnosis of AMI, more specifically NSTEMI to those without any AIRD. Furthermore, patients with RA/SLE/SSC had nearly double the prevalence of anemia and hyperthyroidism, as well as a higher prevalence of chronic pulmonary disease. (Table 1) The median length of stay was also longer in patients with RA/SLE/SSC compared to those without any AIRD (3 vs. 2 days). Furthermore, chronic renal failure was most prevalent amongst patients with SLE (17.7\%) compared to all other groups (9.6-11.7\%). However, rates of previous MI (13.2-14.7\%) and prior PCI (18.7-19.3\%) were similar across all groups, and patients with SSC were less likely to have had prior CABG (4.3\%) than patients without any AIRD (7.4\%).

The crude rates of in-hospital all-cause mortality were higher in patients with SLE and SSC $(1.9 \%$ and $2.4 \%$, respectively) compared to patients with RA and those without any AIRD (1.6\% for both), while all the rheumatological groups (RA/SLE/SSC) were more likely to experience any complication, or bleeding, compared to those without AIRD. (Table 2, Figure 2). The rates of vascular and cardiac complications and stroke were similar between patients with and without AIRD. 
In comparison to patients without AIRD, the odds of adverse events were significantly higher in patients with SSC in multivariate analysis (any complication: OR 1.13 95\% CI 1.01, 1.26; all-cause mortality: OR 1.32 95\% CI 1.03,1.71; bleeding: OR 1.50, 95\% CI 1.30, 1.74; stroke: OR $1.3695 \%$ CI 1.14, 1.62) and were generally not significant in patients with SLE and RA, except reduced odds of all-cause mortality in RA patients (OR 0.79, 95\% CI 0.70, 0.88), and higher odds of bleeding in SLE patients (OR 1.19, 95\% CI 1.09, 1.29). (Table 3, Figure 3).

A subgroup analysis was performed to examine the effect of steroid on outcomes in RA patients. The RA group was stratified into two subgroups depending on documented chronic steroid use (RA-Steroid; $n=4518$ and RA-no steroid: $n=64,836$ ), and the odds of clinical outcomes in the RA-steroid group were assessed in comparison to the RA-no steroid group. Steroid-treated RA patients comprised less than $0.2 \%$ of patients receiving PCI from 20042014, with a much higher use in patients with RA (6.5\%) compared to those without AIRD (0.4\%). Demographic and PCI procedural variables were overall similar between the two groups. (Supplementary Table S2, available at Rheumatology online) However, a more severe clinical presentation phenotype was present for patients with RA on steroid treatment receiving PCI, with a greater percentage of those patients diagnosed with AMI, STEMI, NSTEMI, and shock. Patients with RA on steroids were more likely to undergo multivessel PCI compared to all other groups. RA patients on steroids had a lower prevalence of diabetes (27.6\%), compared to RA patients without steroids (31.2\%) and those without any AIRD (33.4\%).

There was no difference in the crude rates of all-cause mortality, vascular complications and any complication between RA patients with or without chronic steroid use. (Supplementary Table S3, available at Rheumatology online) The crude rates of bleeding and cardiac complications were higher in the RA-steroid group compared to the RA-no steroid group while the rate of stroke was lower in the RA-steroid group. (Supplementary Table S3, available at 
Rheumatology online) There was no difference in the odds of any complication between both RA groups (with and without steroids) and patients without any AIRD or chronic steroid use. (Supplementary Table S4, available at Rheumatology online). However, RA patients with longterm steroid use were associated with reduced odds of all-cause mortality (aOR $0.6995 \% \mathrm{CI}$ 0.52, 0.90), stroke (aOR 0.59 95\% CI 0.47, 0.73) and vascular complications (aOR 0.72 95\% CI $0.52,0.98$ ) and significantly increased odds of bleeding (aOR $1.3595 \%$ CI $1.18,1.55$ ) and cardiac complications (aOR $1.3195 \%$ CI 1.11, 1.56) compared to patients without AIRD. (Supplementary Table S4, available at Rheumatology online, and Figure 3)

\section{Discussion}

The present study of more than 90,000 patients with AIRD is by far the largest to report in-hospital PCI outcomes in this population. We demonstrate that the prevalence of RA amongst patients undergoing PCI has increased over an eleven-year period, while that of other rheumatological diagnoses (SLE and SSC) has remained stable. Our analysis demonstrates significant differences in the in-hospital clinical outcomes, including any complication, allcause mortality, bleeding and stroke after PCI, depending on the subtype of AIRD. Patients with SSC were associated with significantly increased odds of all adverse events, compared to those without AIRD, whereas patients with SLE were at higher risk of bleeding. We observed lower odds of all-cause mortality in patients with RA.

Chronic inflammatory diseases are complex and variable in presentation and treatments, with a spectrum of end-organ effects, including those on the cardiovascular system. Emerging data are uncovering a growing role for cytokine and immune cell regulation of coronary artery and cardiac function, paving the way for future studies in “cardioimmunology".[15] RA, SLE, and SSC augment systemic cytokine signaling of TNF- 
alpha and interleukins 1 and 6, which affect macrophage and monocyte signaling, important cell subtypes in atherosclerotic coronary artery disease.[16] A multitude of factors that predispose to advanced and more severe presentation of CAD in patients with chronic inflammatory diseases including accelerated and more severe atherosclerosis, atypical anginal symptoms that lead to delayed presentation, and increased likelihood of plaque rupture or thrombosis due to severe lesional inflammation.[] $]$ However, these risk factors are not incorporated into traditional cardiovascular risk scores such as the Framingham Risk Score, ASCVD Risk Calculator, or Reynolds Score.[17-19] Furthermore, the current evidence on outcomes of PCI in patients with AIRD is inconsistent and typically limited to small studies. A subgroup analysis of 197 patients with AIRD enrolled in the BASKET-PROVE I (BAsel Stent Kosten-Effektivitäts Trial-PROspective Validation Examination) trial reported increased hazard ratios (HR) of MACE (HR 1.55 95\% CI 1.04, 2.31 ), cardiac death (HR 2.63 95\% CI $1.27,5.43$ ) and all-cause death (HR $2.0595 \%$ CI 1.14, 3.70) in patients with AIRD, compared to those without, over a two year follow-up period.[20] However, this analysis was derived from a highly selected cohort of patients enrolled in this trial (only patients requiring stents $\geq 3 \mathrm{~mm}$ and $<4 \mathrm{~mm}$ in diameter, and excluding any patients with planned surgery within 12 months, those requiring any oral anticoagulation, and those at an increased risk of bleeding) making it less generalizable to the wider population. This analysis examined the effect of allcause AIRD without stratification of outcomes according to the subtype of disease. Furthermore, there is evidence to suggest that patients with AIRD are at a higher risk of ischemic disease. In a single-center cohort study of 936 patients with RA, 32 patients $(3.4 \%)$ underwent PCI for significant CAD and experienced a high rate of MACCE (56\%) over a mean follow-up period of $9( \pm 7)$ years, primarily driven by repeat revascularization $(47 \%)$.[21]

The question of whether RA treatment affects outcomes was evaluated in a singlecenter cohort study of lesions of RA patients matched to lesions within control patients. The 
authors reported that RA predisposes to repeat revascularization at 1 year, with the absence of methotrexate and TNF-alpha inhibitors for RA treatment associated with a $50 \%$ increased relative risk of repeat revascularization following PCI.[13] Patients with AIRD may be less likely to be referred for revascularization due to perception of their high risk of adverse outcomes, which emphasizes the need for outcomes data in this special population.

Analyses of nationwide databases provide a real-world vantage of outcomes on performed procedures, supplementing controlled trials and prospective cohorts and often answering questions on populations excluded from trials or at low prevalence. Yet, even at a low prevalence, conclusions from emerging data on large patient samples provides important information into clinical outcomes from complex groups of patients, providing insight to operators for their decision making processes and assessment of risk. Our present analysis demonstrated a rise in the prevalence of RA amongst patients undergoing PCI over 11 years, although they still remain a minority of patients (1.4\%). Identified RA and other chronic inflammatory diseases are also uncommon in other populations and ethnic groups, evidenced by a recent study of 171,547 hospitalized patients receiving PCI in Taiwan with a $0.31 \%$ and $0.12 \%$ rate of RA and SLE from 2000 to $2010($ RA $n=525$, SLE $n=211)$, respectively.[10] In the Taiwanese study, the authors conclude that RA and SLE were independent predictors of in-hospital all-cause mortality (OR 1.73 95\% CI 1.11, 2.68 and OR 3.81 95\% CI 2.02, 7.16, respectively), and that RA was associated with an increased hazard of ischemic events (HR $1.1895 \%$ CI 1.01, 1.39) and a composite MACE endpoint (HR $1.2095 \%$ CI 1.07, 1.34). However, this analysis was based on a modest number of patients $(<1000)$.

SSC is characterized by obliterative vasculopathy and tissue fibrosis. Peripheral vascular and arterial access is poor in SSC and the vasculature is fibrosed.[22] This may have contributed to our finding of increased risk of post-procedure bleeding in this population. We observed a higher risk of all-cause mortality and stroke in SSC following PCI. This is a novel 
finding and has not be reported before. People with SSS are at an increased risk of stroke and this may be further enhanced in a hypercoagulable post-operative period.[르, 24]

We observed higher odds of post-procedural bleeding in people with SLE. This could be due to thrombocytopenia and platelet dysfunction which are common manifestations of disease activity in SLE.[25] In addition to this, patients with SLE are at increased risk of antiphospholipid antibody syndrome and may be prescribed oral anticoagulants. It is possible that some patients underwent emergency PCI while they were anticoagulated. Finally, hydroxychloroquine, the first line immune suppressive treatment used in people with lupus has been shown to have an anti-thrombotic effect and may contribute to increased risk of postprocedure bleeding.[르]

Our analysis demonstrates differences in mortality and clinical outcomes between patients with and without AIRD. We show that patients with SSC are at a higher adjusted risk of all-cause mortality and complications (any complication, bleeding and stroke) as compared to those without AIRD, which is a significant finding. Although SSC patients have been previously shown to be at a higher risk of AMI, no study to date has reported outcomes in this population.[27] Furthermore, we observe a similarity in outcomes between patients with RA and SLE and those without AIRD, except lower all-cause mortality in patients with RA and higher bleeding risk in those with SLE. The latter findings could be explained by differences in pharmacological regimens that remain unadjusted for in our analysis or other residual confounders. A further possibility to explain the apparent reduced mortality in RA subjects is that further ischemic events may occur in the long-term phase, which is beyond the follow-up duration in our study. Nevertheless, the differences in clinical outcomes between AIRD in a national cohort of PCI hospitalizations provides real-world insight for operators and guides their decision-making when managing this high-risk population that has been previously shown to receive less optimal reperfusion therapy.[2] 
Chronic steroid use in patients with diverse autoimmune diseases has been associated with an increased risk of metabolic syndrome and cardiovascular disease due to its enhancement of plaque formation, arterial stiffness, insulin resistance and lipid levels.[29-31] Perversely, the suppression of inflammation by steroids may also decrease the risk of cardiovascular disease, although there is limited outcomes data to demonstrate this. No study has previously compared outcomes between patients with RA depending on their steroid use. We report lower all-cause mortality and stroke after PCI in patients with RA on steroid therapy, compared to those without AIRD, albeit the expense of higher rates of bleeding and cardiac complications. The lower risk of mortality in patients with RA on steroid therapy may reflect an element of selection bias, whereby only the most stable "healthy" patients are intervened upon, the rest being managed by medical therapy. Our findings emphasize the importance of bleeding-ischemic risk assessment in such patients prior to intervention, and the implementation of bleeding avoidance strategies to minimize the risk of bleeding in patients with chronic steroid use.[32-34]

\section{Limitations}

Our findings need to be interpreted in light of the inherent limitations of large databases. Although the NIS database contained variables of interest, additional data (platelet count, antiplatelet regimen, severity and degree of systemic involvement of AIRD and use of disease modifying drugs such as tumor necrosis factor (TNF) alpha inhibitors, non-steroidal antiinflammatory drugs and methotrexate, and duration of steroid use), the exert and severity of coronary disease and lesion complexity are not routinely collected and may provide additional information to better stratify risk, case complexity, and procedural outcomes. Additionally, NIS only captures in-hospital outcomes and it is possible that longer-term data on mortality and other adverse events such as reinfarction follow a more unfavorable course in patients with 


\begin{abstract}
AIRD. Furthermore, despite the considerable granular data relating to the PCI admission (e.g. STEMI on presentation vs. during hospitalization) in NIS, full procedural details are not recorded, which limits insights into differences in angiographic findings (e.g. target lesion or vessel revascularization and stent thrombosis), PCI procedural techniques (e.g. femoral vs. radial access), and possibly clinical outcomes. Furthermore, the NIS dataset does not capture platelet or hemoglobin counts, which may influence antiplatelet and stent choice. The NIS dataset only captures inpatient PCI procedures and, therefore, our analysis provides no insight into the prevalence and outcomes of AIRD in PCI procedures undertaken in the outpatient setting. Finally, in keeping with all observational registry work, the possibility of unmeasured or unrecognized confounders may contribute to the adverse outcomes, although capture of a wide range of comorbid conditions in the NIS may help to mitigate this bias.
\end{abstract}

\title{
Conclusion
}

In a national analysis of PCI hospitalizations over an eleven-year period, we demonstrate differences in in-hospital outcomes of patients with AIRD according to disease subtype, with SSC patients being at the highest risk of mortality and adverse outcomes. Our findings provide operators with further insight into the clinical outcomes of this complex riskgroup with an inherently high risk of progressive coronary artery disease, and support a multidisciplinary strategy in managing these patients involving rheumatologists.

\section{Conflicts}

The authors have no disclosures and no relationships with the pharmaceutical industry. 


\section{Funding}

Dr Mohamed Mohamed receives PhD funding from Medtronic Ltd. Medtronic Ltd. was not involved in the conceptualization, design, conduct, analysis, or interpretation of the current study.

\section{Statement}

The manuscript has neither been published nor is currently under consideration for publication by any other journal. All authors have approved the final version of the manuscript.

\section{References}

1 Lindhardsen J, Ahlehoff O, Gislason GH, et al. The risk of myocardial infarction in rheumatoid arthritis and diabetes mellitus: a Danish nationwide cohort study. Annals of the Rheumatic Diseases 2011;70(6):929.

2 Dregan A, Charlton J, Chowienczyk P, Gulliford MC. Chronic inflammatory disorders and risk of type 2 diabetes mellitus, coronary heart disease, and stroke: a population-based cohort study. Circulation 2014;130(10):837-44.

3 Ali $\mathrm{H}, \mathrm{Ng} \mathrm{KR}$, Low AHL. A qualitative systematic review of the prevalence of coronary artery disease in systemic sclerosis. International Journal of Rheumatic Diseases 2015;18(3):276-86.

4 Murphy SL, Xu J, Kochanek KD, Arias E. Mortality in the United States, 2017. NCHS Data Brief 2018(328):1-8.

5 Gabriel SE, Michaud K. Epidemiological studies in incidence, prevalence, mortality, and comorbidity of the rheumatic diseases. Arthritis Res Ther 2009;11(3):229.

6 Avina-Zubieta JA, Thomas J, Sadatsafavi M, Lehman AJ, Lacaille D. Risk of incident cardiovascular events in patients with rheumatoid arthritis: a meta-analysis of observational studies. Ann Rheum Dis 2012;71(9):1524-9.

7 Mantel A, Holmqvist M, Jernberg T, Wallberg-Jonsson S, Askling J. Rheumatoid arthritis is associated with a more severe presentation of acute coronary syndrome and worse short-term outcome. Eur Heart J 2015;36(48):3413-22.

8 Mason JC, Libby P. Cardiovascular disease in patients with chronic inflammation: mechanisms underlying premature cardiovascular events in rheumatologic conditions. Eur Heart J 2015;36(8):482-9c.

9 Maksimowicz-McKinnon K, Selzer F, Manzi S, et al. Poor 1-year outcomes after percutaneous coronary interventions in systemic lupus erythematosus: report from the National Heart, Lung, and Blood Institute Dynamic Registry. Circ Cardiovasc Interv 2008;1(3):201-8. 10 Lai CH, Lai WW, Chiou MJ, et al. Outcomes of percutaneous coronary intervention in patients with rheumatoid arthritis and systemic lupus erythematosus: an 11-year nationwide cohort study. Ann Rheum Dis 2016;75(7):1350-6.

11 Tarek el G, Yasser AE, Gheita T. Coronary angiographic findings in asymptomatic 
systemic sclerosis. Clin Rheumatol 2006;25(4):487-90.

12 Akram MR, Handler CE, Williams M, et al. Angiographically proven coronary artery disease in scleroderma. Rheumatology (Oxford) 2006;45(11):1395-8.

13 Sintek MA, Sparrow CT, Mikuls TR, et al. Repeat revascularisation outcomes after percutaneous coronary intervention in patients with rheumatoid arthritis. Heart 2016;102(5):363.

14 Agency for Healthcare Research and Quality R, MD. HCUP NIS Database Documentation. Healthcare Cost and Utilization Project (HCUP). In; February 2018.

15 Swirski FK, Nahrendorf M. Cardioimmunology: the immune system in cardiac homeostasis and disease. Nat Rev Immunol 2018;18(12):733-44.

16 Niccoli G, Montone RA, Sabato V, Crea F. Role of Allergic Inflammatory Cells in Coronary Artery Disease. Circulation 2018;138(16):1736-48.

17 Wilson PW, D'Agostino RB, Levy D, Belanger AM, Silbershatz H, Kannel WB. Prediction of coronary heart disease using risk factor categories. Circulation 1998;97(18):1837-47.

18 Goff DC, Jr., Lloyd-Jones DM, Bennett G, et al. 2013 ACC/AHA guideline on the assessment of cardiovascular risk: a report of the American College of Cardiology/American Heart Association Task Force on Practice Guidelines. Circulation 2014;129(25 Suppl 2):S4973.

19 Ridker PM, Buring JE, Rifai N, Cook NR. Development and validation of improved algorithms for the assessment of global cardiovascular risk in women: the Reynolds Risk Score. JAMA 2007;297(6):611-9.

20 Nochioka K, Biering-Sorensen T, Hansen KW, et al. Long-term outcomes in patients with rheumatologic disorders undergoing percutaneous coronary intervention: a BAsel Stent Kosten-Effektivitats Trial-PROspective Validation Examination (BASKET-PROVE) substudy. Eur Heart J Acute Cardiovasc Care 2017;6(8):778-86.

21 Spartera M, Godino C, Baldissera E, et al. Long-term clinical outcomes of patients with rheumatoid arthritis and concomitant coronary artery disease. Am J Cardiovasc Dis 2017;7(1):9-18.

22 Foocharoen C, Pussadhamma B, Mahakkanukrauh A, Suwannaroj S, Nanagara R. Asymptomatic cardiac involvement in Thai systemic sclerosis: prevalence and clinical correlations with non-cardiac manifestations (preliminary report). Rheumatology (Oxford) 2015;54(9):1616-21.

23 Bissell LA, Dumitru RB, Erhayiem B, et al. Incidental significant arrhythmia in scleroderma associates with cardiac magnetic resonance measure of fibrosis and hs-TnI and NT-proBNP. Rheumatology (Oxford) 2019;58(7):1221-6.

24 Vacca A, Meune C, Gordon J, et al. Cardiac arrhythmias and conduction defects in systemic sclerosis. Rheumatology (Oxford) 2014;53(7):1172-7.

25 Fayyaz A, Igoe A, Kurien BT, et al. Haematological manifestations of lupus. Lupus Sci Med 2015;2(1):e000078.

26 Petri M. Use of hydroxychloroquine to prevent thrombosis in systemic lupus erythematosus and in antiphospholipid antibody-positive patients. Curr Rheumatol Rep 2011;13(1):77-80.

27 Chu S-Y, Chen Y-J, Liu C-J, et al. Increased Risk of Acute Myocardial Infarction in Systemic Sclerosis: A Nationwide Population-based Study. The American Journal of Medicine 2013;126(11):982-8.

28 Van Doornum S, Brand C, Sundararajan V, Ajani AE, Wicks IP. Rheumatoid arthritis patients receive less frequent acute reperfusion and secondary prevention therapy after myocardial infarction compared with the general population. Arthritis Res Ther 2010;12(5):R183. 
29 de Oliveira BMGB, Medeiros MMdC, de Cerqueira JVM, de Souza Quixadá RT, de Oliveira ÍMAX. Metabolic syndrome in patients with rheumatoid arthritis followed at a University Hospital in Northeastern Brazil. Revista Brasileira de Reumatologia (English Edition) 2016;56(2):117-25.

30 Dessein PH, Joffe BI, Stanwix AE, Christian BF, Veller M. Glucocorticoids and insulin sensitivity in rheumatoid arthritis. J Rheumatol 2004;31(5):867-74.

31 Hafstrom I, Rohani M, Deneberg S, Wornert M, Jogestrand T, Frostegard J. Effects of low-dose prednisolone on endothelial function, atherosclerosis, and traditional risk factors for atherosclerosis in patients with rheumatoid arthritis--a randomized study. J Rheumatol 2007;34(9):1810-6.

32 Mohamed MO, Kinnaird T, Anderson R, et al. Combinations of bleeding and ischemic risk and their association with clinical outcomes in acute coronary syndrome. International Journal of Cardiology 2019;290:7-14.

33 Kwok CS, Rao SV, Myint PK, et al. Major bleeding after percutaneous coronary intervention and risk of subsequent mortality: a systematic review and meta-analysis. Open Heart 2014;1(1).

34 Desai NR, Peterson ED, Chen AY, et al. Balancing the risk of mortality and major bleeding in the treatment of NSTEMI patients - A report from the National Cardiovascular Data Registry. American Heart Journal 2013;166(6):1043-9.e1.

\section{Figures and legends:}

Figure 1: Prevalence of study groups amongst PCI hospitalizations over the study years.

Footnote: RA: Rheumatoid arthritis; SLE: Systemic lupus erythematosus; SSC: Scleroderma; Trends: $\mathrm{p}<0.001$ for RA, $\mathrm{p}=0.09$ for SLE and $\mathrm{p}=0.34$ for SSC.

Figure 2. In-hospital adverse events of study groups

Footnote: AIRD: autoimmune rheumatic disease; RA: Rheumatoid arthritis; SLE: Systemic lupus erythematosus; SSC: Scleroderma.

\section{Figure 3. Adjusted odds ratios (aOR) of adverse outcomes in rheumatological disease groups*}

Footnote: *Reference group for each outcome is patients without autoimmune rheumatic disease; RA: Rheumatoid arthritis; SLE: Systemic lupus erythematosus; SSC: Scleroderma. 
Figure 1: Prevalence of study groups amongst PCI hospitalizations over the study years. Footnote: RA: Rheumatoid arthritis; SLE: Systemic lupus erythematosus; SSC: Scleroderma; Trends: $p<0.001$ for RA, $p=0.09$ for SLE and $p=0.34$ for SSC.

$50 \times 30 \mathrm{~mm}(300 \times 300$ DPI $)$ 
Caption : Figure 2. In-hospital adverse events of study groups

Footnote: AIRD: autoimmune rheumatic disease; RA: Rheumatoid arthritis; SLE: Systemic lupus erythematosus; SSC: Scleroderma. 
Figure 3. Adjusted odds ratios (aOR) of adverse outcomes in rheumatological disease groups*

Footnote: *Reference group for each outcome is patients without autoimmune rheumatic disease; RA: Rheumatoid arthritis; SLE: Systemic lupus erythematosus; SSC: Scleroderma.

$18 \times 24 \mathrm{~mm}(300 \times 300 \mathrm{DPI})$ 
Table 1: Patient demographics and procedural characteristics of study groups

\begin{tabular}{|c|c|c|c|c|}
\hline Patient Characteristics & $\begin{array}{c}\text { No AIRD } \\
(\mathrm{n}=6,468,478)\end{array}$ & $\begin{array}{c}\text { RA } \\
(n=69,354)\end{array}$ & $\begin{array}{c}\text { SLE } \\
(\mathrm{n}=17,661)\end{array}$ & $\begin{array}{c}\text { SSC } \\
(n=3454)\end{array}$ \\
\hline Age, years, median (IQR) & $65(56,74)$ & $68(60,76)$ & $60(51,69)$ & $67(58,73)$ \\
\hline Males & $66.7 \%$ & $44.2 \%$ & $21.9 \%$ & $25.7 \%$ \\
\hline White & $63.1 \%$ & $67.8 \%$ & $56.8 \%$ & $67.8 \%$ \\
\hline Black & $6.3 \%$ & $5.7 \%$ & $15.5 \%$ & $5.8 \%$ \\
\hline Hispanic & $5.3 \%$ & $3.9 \%$ & $6.4 \%$ & $3.6 \%$ \\
\hline Asian/Pacific Islander & $1.7 \%$ & $1.1 \%$ & $1.1 \%$ & $1.2 \%$ \\
\hline Native American & $0.4 \%$ & $0.4 \%$ & $0.4 \%$ & $0.7 \%$ \\
\hline Other & $2.9 \%$ & $2.2 \%$ & $2.3 \%$ & $2.6 \%$ \\
\hline Missing Ethnicity & $20.1 \%$ & $18.8 \%$ & $17.5 \%$ & $18.3 \%$ \\
\hline Admission types - Elective & $27.2 \%$ & $22.4 \%$ & $22.2 \%$ & $24.3 \%$ \\
\hline Admission Day, Weekday & $84.0 \%$ & $82.2 \%$ & $81.5 \%$ & $83.5 \%$ \\
\hline \multicolumn{5}{|l|}{ Median ZIP income } \\
\hline 1st quartile & $26.3 \%$ & $26.4 \%$ & $30.5 \%$ & $23.3 \%$ \\
\hline 2nd quartile & $26.8 \%$ & $28.0 \%$ & $26.2 \%$ & $24.5 \%$ \\
\hline 3rd quartile & $26.8 \%$ & $25.0 \%$ & $23.3 \%$ & $24.5 \%$ \\
\hline 4th quartile & $22.1 \%$ & $20.6 \%$ & $20.0 \%$ & $27.6 \%$ \\
\hline \multicolumn{5}{|l|}{ Expected Primary Payer } \\
\hline Medicare & $51.2 \%$ & $65.4 \%$ & $53.3 \%$ & $53.3 \%$ \\
\hline Medicaid & $5.8 \%$ & $4.8 \%$ & $9.4 \%$ & $4.3 \%$ \\
\hline Private & $34.7 \%$ & $25.9 \%$ & $32.0 \%$ & $30.3 \%$ \\
\hline Uninsured & $5.0 \%$ & $2.0 \%$ & $3.0 \%$ & $0.7 \%$ \\
\hline No charge & $0.5 \%$ & $0.2 \%$ & $0.3 \%$ & $0.1 \%$ \\
\hline Other & $2.8 \%$ & $1.8 \%$ & $2.0 \%$ & $1.2 \%$ \\
\hline \multicolumn{5}{|l|}{ Procedure Details } \\
\hline Single vessel PCI & $72.9 \%$ & $73.5 \%$ & $74.3 \%$ & $75.7 \%$ \\
\hline Multi-vessel PCI & $17.9 \%$ & $17.4 \%$ & $16.8 \%$ & $14.4 \%$ \\
\hline Unknown vessel number & $9.2 \%$ & $9.1 \%$ & $8.9 \%$ & $9.9 \%$ \\
\hline Bifurcation stenting & $1.7 \%$ & $1.8 \%$ & $1.5 \%$ & $2.1 \%$ \\
\hline Use of assist devise or IABP & $3.3 \%$ & $3.3 \%$ & $3.1 \%$ & $4.1 \%$ \\
\hline Bare Metal Stent & $22.0 \%$ & $23.5 \%$ & $23.6 \%$ & $25.0 \%$ \\
\hline Drug Eluting Stent & $73.3 \%$ & $71.5 \%$ & $69.7 \%$ & $70.6 \%$ \\
\hline Unknown Stent Type & $7.0 \%$ & $7.2 \%$ & $8.2 \%$ & $6.6 \%$ \\
\hline Both stent types used & $2.3 \%$ & $2.2 \%$ & $1.5 \%$ & $2.2 \%$ \\
\hline Fractional flow reserve & $0.7 \%$ & $1.1 \%$ & $1.2 \%$ & $1.3 \%$ \\
\hline Intravascular ultrasound & $4.9 \%$ & $5.4 \%$ & $5.3 \%$ & $4.9 \%$ \\
\hline \multicolumn{5}{|l|}{ Record Characteristics } \\
\hline Primary diagnosis AMI & $41.0 \%$ & $45.6 \%$ & $43.3 \%$ & $42.9 \%$ \\
\hline STEMI & $22.9 \%$ & $23.4 \%$ & $22.2 \%$ & $21.1 \%$ \\
\hline NSTEMI & $22.8 \%$ & $27.7 \%$ & $26.4 \%$ & $27.4 \%$ \\
\hline Diagnosis of Shock & $2.8 \%$ & $2.9 \%$ & $3.1 \%$ & $3.3 \%$ \\
\hline $\begin{array}{l}\text { Length of stay, days, median } \\
\text { (IQR) }\end{array}$ & $2(1,4)$ & $3(1,4)$ & $3(2,5)$ & $3(1,5)$ \\
\hline
\end{tabular}




\begin{tabular}{|c|c|c|c|c|}
\hline $\begin{array}{l}\text { Total charge, \$, median } \\
\text { (IQR) }\end{array}$ & $\begin{array}{c}\$ 17,434 \\
(\$ 12,996, \\
\$ 24,256)\end{array}$ & $\begin{array}{c}\$ 18,258 \\
(\$ 13,641, \\
\$ 25,304)\end{array}$ & $\begin{array}{c}\$ 18,277 \\
(\$ 13,640, \\
\$ 25,568)\end{array}$ & $\begin{array}{r}\$ 18,401 \\
(\$ 13,661, \\
\$ 26,551)\end{array}$ \\
\hline \multicolumn{5}{|l|}{ Comorbidities } \\
\hline Hypercholesterolemia & $13.5 \%$ & $11.2 \%$ & $10.8 \%$ & $8.1 \%$ \\
\hline Smoking & $35.5 \%$ & $35.0 \%$ & $33.4 \%$ & $24.9 \%$ \\
\hline AIDS & $0.01 \%$ & $0.01 \%$ & $0.01 \%$ & $0 \%$ \\
\hline Alcohol abuse & $2.0 \%$ & $1.2 \%$ & $1.2 \%$ & $0.9 \%$ \\
\hline Anemia & $8.6 \%$ & $16.5 \%$ & $18.7 \%$ & $19.3 \%$ \\
\hline Congestive heart failure & $0.9 \%$ & $1.4 \%$ & $1.4 \%$ & $3.1 \%$ \\
\hline Chronic Pulmonary disease & $15.4 \%$ & $23.6 \%$ & $21.7 \%$ & $17.3 \%$ \\
\hline Coagulopathy & $2.2 \%$ & $2.7 \%$ & $5.3 \%$ & $2.0 \%$ \\
\hline Depression & $5.3 \%$ & $8.6 \%$ & $10.1 \%$ & $7.8 \%$ \\
\hline Diabetes & $33.4 \%$ & $31.0 \%$ & $26.5 \%$ & $21.2 \%$ \\
\hline Drug abuse & $1.3 \%$ & $0.1 \%$ & $1.9 \%$ & $1.2 \%$ \\
\hline Hypertension & $69.7 \%$ & $72.6 \%$ & $70.8 \%$ & $64.0 \%$ \\
\hline Hyperthyroidism & $7.8 \%$ & $13.9 \%$ & $14.6 \%$ & $19.2 \%$ \\
\hline Liver disease & $0.1 \%$ & $0.1 \%$ & $1.5 \%$ & $2.6 \%$ \\
\hline Lymphoma & $0.3 \%$ & $0.4 \%$ & $0.5 \%$ & $0.5 \%$ \\
\hline Fluid \& electrolyte disorders & $9.3 \%$ & $12.9 \%$ & $13.7 \%$ & $13.7 \%$ \\
\hline Other Neurological disorders & $2.95 \%$ & $3.8 \%$ & $5.7 \%$ & $3.5 \%$ \\
\hline Obesity & $12.2 \%$ & $12.1 \%$ & $11.5 \%$ & $5.5 \%$ \\
\hline Paralysis & $0.7 \%$ & $0.7 \%$ & $1.0 \%$ & $0.1 \%$ \\
\hline Peripheral vascular disorder & $10.3 \%$ & $11.7 \%$ & $9.8 \%$ & $13.9 \%$ \\
\hline Psychoses & $1.3 \%$ & $1.5 \%$ & $2.1 \%$ & $0.8 \%$ \\
\hline $\begin{array}{l}\text { Pulmonary circulation } \\
\text { disorders }\end{array}$ & $0.2 \%$ & $0.3 \%$ & $0.3 \%$ & $1.9 \%$ \\
\hline Renal Failure & $9.6 \%$ & $10.7 \%$ & $17.7 \%$ & $11.7 \%$ \\
\hline $\begin{array}{l}\text { Peptic ulcer disease without } \\
\text { bleeding }\end{array}$ & $0.03 \%$ & $0.08 \%$ & $0.03 \%$ & $0.03 \%$ \\
\hline Valvular disease & $0.3 \%$ & $0.5 \%$ & $0.7 \%$ & $0.9 \%$ \\
\hline Weight loss & $0.1 \%$ & $1.3 \%$ & $1.4 \%$ & $2.1 \%$ \\
\hline Metastatic cancer & $0.3 \%$ & $0.3 \%$ & $0.2 \%$ & $0.1 \%$ \\
\hline Previous MI & $13.2 \%$ & $13.8 \%$ & $13.8 \%$ & $14.7 \%$ \\
\hline Previous PCI & $18.9 \%$ & $18.7 \%$ & $18.5 \%$ & $19.3 \%$ \\
\hline Previous CABG & $7.4 \%$ & $6.1 \%$ & $5.4 \%$ & $4.3 \%$ \\
\hline Previous CVA & $3.8 \%$ & $4.9 \%$ & $6.3 \%$ & $4.4 \%$ \\
\hline
\end{tabular}

AIRD: rheumatological diagnosis; RA: Rheumatoid arthritis; SLE: Systemic Lupus Erythematosus; SSC: Systemic sclerosis; AMI: acute myocardial infarction; CABG: coronary artery bypass graft; CAD: coronary artery disease; CVA: cerebrovascular accident (stroke or transient ischemic attack); IHD: ischemic heart disease; IQR: interquartile range; NSTEMI: non-ST-elevation myocardial infarction; PCI: percutaneous coronary intervention; STEMI: ST-Elevation myocardial infarction 
Table 2: In-hospital clinical outcomes

\begin{tabular}{|l|c|c|c|c|}
\hline & $\begin{array}{c}\text { No AIRD } \\
(\mathrm{n}=6,468,478)\end{array}$ & $\begin{array}{c}\text { RA } \\
(\mathrm{n}=69,354)\end{array}$ & $\begin{array}{c}\text { SLE } \\
(\mathrm{n}=17,661)\end{array}$ & $\begin{array}{c}\text { SSC } \\
(\mathrm{n}=3454)\end{array}$ \\
\hline Any complication & $9.0 \%$ & $11.6 \%$ & $12.3 \%$ & $13.6 \%$ \\
\hline Mortality & $1.6 \%$ & $1.6 \%$ & $1.9 \%$ & $2.4 \%$ \\
\hline Bleeding & $3.3 \%$ & $5.7 \%$ & $6.4 \%$ & $8.1 \%$ \\
\hline $\begin{array}{l}\text { Vascular } \\
\text { complication }\end{array}$ & $1.0 \%$ & $1.0 \%$ & $1.4 \%$ & $1.0 \%$ \\
\hline $\begin{array}{l}\text { Cardiac } \\
\text { complication }\end{array}$ & $3.0 \%$ & $3.1 \%$ & $3.1 \%$ & $2.5 \%$ \\
\hline Stroke & $2.9 \%$ & $3.4 \%$ & $2.7 \%$ & $4.4 \%$ \\
\hline
\end{tabular}

AIRD: rheumatological diagnosis; RA: Rheumatoid arthritis; SLE: Systemic Lupus Erythematosus; SSC: Systemic sclerosis 
Table 3: Adjusted odds ratios (OR) of in-hospital adverse outcomes for each disease type*

\begin{tabular}{|l|c|c|c|c|c|c|}
\hline \multirow{2}{*}{} & \multicolumn{2}{|c|}{ RA } & \multicolumn{2}{c|}{ SLE } & \multicolumn{2}{c|}{ SSC } \\
\cline { 2 - 7 } & OR $(95 \% \mathrm{CI})$ & $\begin{array}{c}\mathrm{p}- \\
\text { value }\end{array}$ & OR $(95 \% \mathrm{CI})$ & $\begin{array}{c}\mathrm{p}- \\
\text { value }\end{array}$ & OR $(95 \% \mathrm{CI})$ & $\begin{array}{c}\mathrm{p}- \\
\text { value }\end{array}$ \\
\hline $\begin{array}{l}\text { Any } \\
\text { complication }\end{array}$ & $0.97(0.93,1.02)$ & 0.216 & $1.06(0.99,1.13)$ & 0.067 & $1.13(1.01,1.26)$ & 0.036 \\
\hline Mortality & $0.79(0.70,0.88)$ & $<0.001$ & $1.02(0.87,1.19)$ & 0.853 & $1.32(1.03,1.71)$ & 0.030 \\
\hline Bleeding & $1.06(0.99,1.14)$ & 0.068 & $1.19(1.09,1.29)$ & $<0.001$ & $1.50(1.30,1.74)$ & $<0.001$ \\
\hline Stroke & $0.95(0.88,1.03)$ & 0.205 & $0.91(0.82,1.02)$ & 0.115 & $1.36(1.14,1.62)$ & 0.001 \\
\hline
\end{tabular}

*reference is patients without autoimmune rheumatological diagnosis; CI: confidence interval; RA: Rheumatoid arthritis; SLE: Systemic Lupus Erythematosus; SSC: Systemic sclerosis 
Supplementary table S1: ICD-9-CM codes for complications

\begin{tabular}{|l|l|}
\hline Post-procedural Complication & ICD-9-CM codes \\
\hline Bleeding complications & \\
\hline Gastrointestinal & 578.9 \\
\hline Unspecified haemorrhage & 459.0 \\
\hline Retroperitoneal haemorrhage & 568.81 \\
\hline Intracranial haemorrhage & 432.9 \\
\hline Intracerebral haemorrhage & $431 . x$ \\
\hline Blood transfusion & V58.2, 99.0x (procedure) \\
\hline Post-op haemorrhage requiring transfusion & 99.0 (procedure) \\
\hline Vascular complications & \\
\hline Vascular injury & $900-904,998.2,447,868.04$ (diagnosis) \\
\hline Cardiac complications & $39.31,39.41,39.49,39.52,39.53,39.56$ - 39.59 \\
\hline Iatrogenic cardiac & 39.79 (procedure) \\
\hline Pericardial comp & 997.1 \\
\hline Requiring CABG & $423.0,423.3$ (diagnosis) 47.0 (procedure) \\
\hline Stroke/TIA & $36.1 x, 36.2,36.31,36.32,36.9 x$ \\
\hline
\end{tabular}


Supplementary Table S2. Rheumatoid arthritis patient characteristics stratified by long term use of steroids

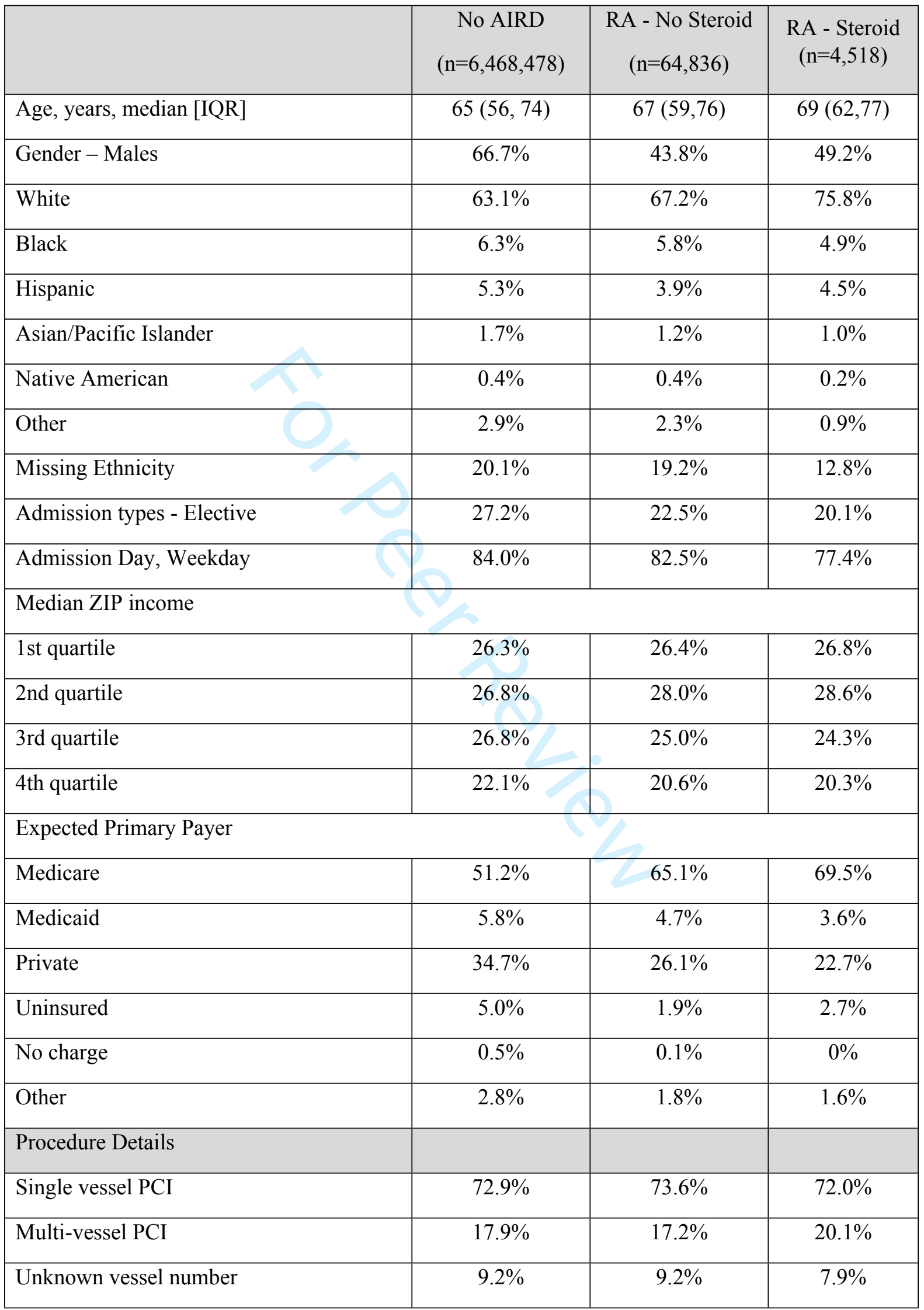




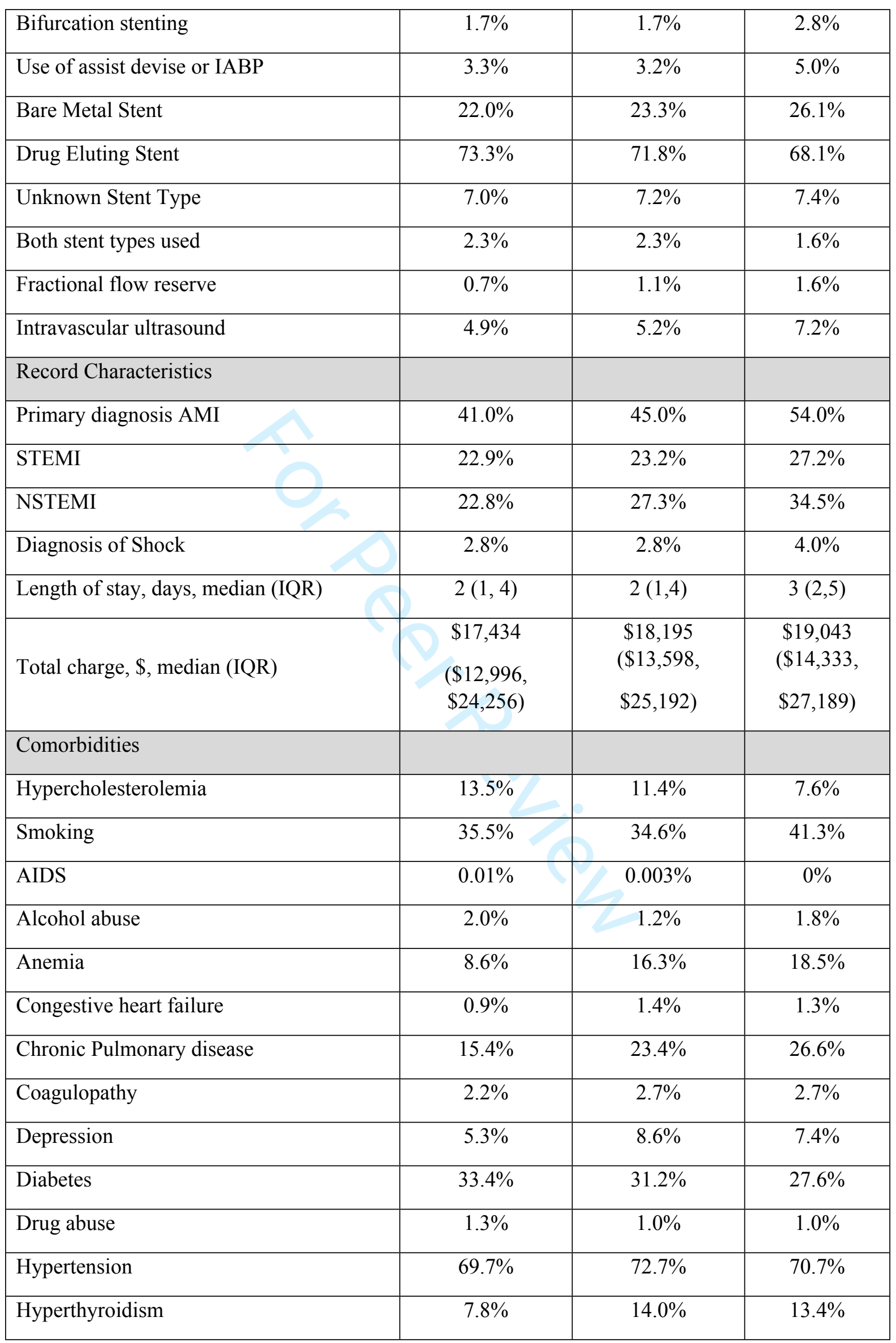




\begin{tabular}{|c|c|c|c|}
\hline Liver disease & $0.1 \%$ & $0.9 \%$ & $0.9 \%$ \\
\hline Lymphoma & $0.3 \%$ & $0.4 \%$ & $0.3 \%$ \\
\hline Fluid \& electrolyte disorders & $9.3 \%$ & $12.6 \%$ & $16.6 \%$ \\
\hline Other Neurological disorders & $2.95 \%$ & $3.8 \%$ & $3.1 \%$ \\
\hline Obesity & $12.2 \%$ & $12.1 \%$ & $11.6 \%$ \\
\hline Paralysis & $0.7 \%$ & $0.7 \%$ & $0.2 \%$ \\
\hline Peripheral vascular disorder & $10.3 \%$ & $11.7 \%$ & $11.9 \%$ \\
\hline Psychoses & $1.3 \%$ & $1.6 \%$ & $0.7 \%$ \\
\hline Pulmonary circulation disorders & $0.2 \%$ & $0.3 \%$ & $0.6 \%$ \\
\hline Renal Failure & $9.6 \%$ & $10.6 \%$ & $12.0 \%$ \\
\hline Peptic ulcer disease excluding bleeding & $0.03 \%$ & $0.01 \%$ & $0 \%$ \\
\hline Valvular disease & $0.3 \%$ & $0.5 \%$ & $0.1 \%$ \\
\hline Weight loss & $0.1 \%$ & $1.3 \%$ & $1.3 \%$ \\
\hline Metastatic cancer & $0.3 \%$ & $0.03 \%$ & $0.03 \%$ \\
\hline Previous MI & $13.2 \%$ & $13.8 \%$ & $14.1 \%$ \\
\hline Previous PCI & $18.9 \%$ & $18.5 \%$ & $21.7 \%$ \\
\hline Previous CABG & $7.4 \%$ & $6.2 \%$ & $5.2 \%$ \\
\hline Previous CVA & $3.8 \%$ & $4.8 \%$ & $6.7 \%$ \\
\hline
\end{tabular}

AIRD: rheumatological diagnosis; RA: Rheumatoid arthritis; SLE: Systemic Lupus Erythematosus; SSC: Systemic sclerosis; AMI: acute myocardial infarction; CABG: coronary artery bypass graft; CAD: coronary artery disease; CVA: cerebrovascular accident (stroke or transient ischemic attack); IHD: ischemic heart disease; IQR: interquartile range; NSTEMI: non-ST-elevation myocardial infarction; PCI: percutaneous coronary intervention; STEMI: ST-Elevation myocardial infarction Supplementary Table S3. Rheumatoid arthritis patient complications stratified by long term steroid use

\begin{tabular}{|l|c|c|c|c|}
\hline \multicolumn{1}{|c|}{ Outcome } & $\begin{array}{c}\text { No AIRD } \\
(\mathrm{n}=6,468,478)\end{array}$ & $\begin{array}{c}\text { RA - No Steroid } \\
(\mathrm{n}=64,836)\end{array}$ & $\begin{array}{c}\text { RA - Steroid } \\
(\mathrm{n}=4,518)\end{array}$ & $\mathrm{p}$-value \\
\hline Any complication & $9.0 \%$ & $11.5 \%$ & $12.5 \%$ & 0.062 \\
\hline Mortality & $1.6 \%$ & $1.6 \%$ & $1.6 \%$ & 0.757 \\
\hline Bleeding & $3.3 \%$ & $5.5 \%$ & $7.3 \%$ & $<0.001$ \\
\hline Vascular complications & $1.0 \%$ & $1.1 \%$ & $1.0 \%$ & 0.511 \\
\hline Cardiac complications & $3.0 \%$ & $3.1 \%$ & $4.2 \%$ & $<0.001$ \\
\hline Stroke & $2.9 \%$ & $3.5 \%$ & $2.1 \%$ & $<0.001$ \\
\hline
\end{tabular}


AIRD: autoimmune rheumatic disease; RA: rheumatoid arthritis

Supplementary Table S4. Adjusted odds ratios (OR) of in-hospital adverse events in patients with rheumatoid arthritis (RA), stratified by steroid use*

\begin{tabular}{|l|c|c|c|c|}
\hline & RA-no steroids* & & RA-steroids* & \\
\hline Outcome & OR $(95 \% \mathrm{CI})$ & $\mathrm{p}$-value & OR $(95 \% \mathrm{CI})$ & $\mathrm{p}$-value \\
\hline Any complication & $0.96[0.91,1.01]$ & 0.099 & $0.99(0.89,1.09)$ & 0.803 \\
\hline Mortality & $0.84[0.74,0.94]$ & 0.003 & $0.69(0.52,0.90)$ & 0.007 \\
\hline Bleeding & $1.03[0.96,1.10]$ & 0.433 & $1.35(1.18,1.55)$ & $<0.001$ \\
\hline $\begin{array}{l}\text { Vascular } \\
\text { complications }\end{array}$ & $0.79[0.69,0.91]$ & 0.001 & $0.72(0.52,0.98)$ & 0.047 \\
\hline Cardiac complications & $1.02[0.93,1.12]$ & 0.696 & $1.31(1.11,1.56)$ & 0.002 \\
\hline Stroke & $0.99[0.91,1.07]$ & 0.761 & $0.59(0.47,0.73)$ & $<0.001$ \\
\hline
\end{tabular}

*reference group: patients without autoimmune rheumatic disease or steroid use; CI: Confidence interval; RA: rheumatoid arthritis. 